\title{
Spectra of heavy quarkonia in a Bethe-Salpeter-equation approach
}

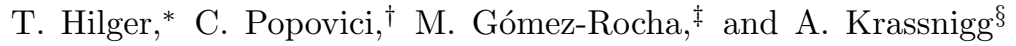 \\ University of Graz, Institute of Physics, NAWI Graz, A-8010 Graz, Austria
}

(Dated: August 13, 2018)

\begin{abstract}
In a covariant Bethe-Salpeter-equation approach and with a rainbow-ladder truncated model of QCD, we investigate the use of an effective interaction with the goal of reproducing QCD phenomenology. We extend previous studies and present results for ground and excited meson states in the bottomonium and charmonium systems, where the results are surprisingly good for most states. In addition, we formulate a critical outlook on states with exotic quantum numbers as well as the light-quark domain.
\end{abstract}

PACS numbers: 14.40.-n, 12.38.Lg, 11.10.St

\section{INTRODUCTION}

One of the challenges of modern standard-model particle physics is the description of mesons and baryons via the fundamental degrees of freedom in quantum chromodynamics (QCD), quarks and gluons. The stronginteraction sector of the standard model is beautifully accessible via the asymptotic freedom of QCD [1-3, but the low-energy properties of hadrons and most prominently confinement and dynamical chiral symmetry breaking $(\mathrm{D} \chi \mathrm{SB})$ are accessible from the underlying quantum field theory (QFT) only by nonperturbative methods; in addition, a thorough understanding of these phenomena is paramount for theoretical hadron physics 4 .

The recent renaissance of hadron spectroscopy, in particular, is due to the fact that this field still offers immediate and influential open question, e.g., the existence, properties, and abundance of hadron states with exotic quantum numbers. Any modern comprehensive approach to hadron spectroscopy must therefore go beyond the conventional states described by the quark model -in the meson sector by a standard quark-antiquark $(q \bar{q})$ configuration - and address these open problems.

Modern approaches to hadron spectroscopy make use of lattice-regularized QCD techniques on one hand [5] 7], and continuum QFT methods on the other 8 11] (always see also references therein). Our method of choice in the present work is the coupled Dyson-Schwinger-BetheSalpeter-equation (DSBSE) framework, which has been successfully applied not only to QCD but also to other strongly coupled theories, such as $\mathrm{QED}_{3}$ or graphene; see for example 12 14 for recent reviews.

The DSBSE studies of the past decades have been undertaken at varying levels of sophistication. Only in a few particular cases analytical solutions are accessible, such as the limit of heavy quark mass, where the system can be described by a variant of heavy-quark effec-

\footnotetext{
* thomas.hilger@uni-graz.at

$\dagger$ carina.popovici@uni-graz.at

$\ddagger$ maria.gomez-rocha@uni-graz.at

$\S \overline{\text { andreas.krassnigg@uni-graz.at }}$
}

tive theory 15, or if only the IR behavior of the theory is considered [16]. All other studies, and ours as well, rely on truncations that enable numerical investigations. The infinite tower of coupled DSEs is truncated by restricting the number of equations that are solved self-consistently, and by compensating for the remaining equations through sound Ansätze for the corresponding Green functions that are not taken into account explicitly.

In particular, we use a basic but symmetry-preserving truncation to study mesons by solving the quark DysonSchwinger equation (DSE) coupled to the meson $q \bar{q}$ Bethe-Salpeter equation (BSE). Baryon studies are not performed in the present work, but such studies can be carried out on an equally consistent footing using covariant quark-diquark or three-quark-equation setups, see e. g., [17 24] and references therein for details.

Despite the difficulty inherent to nonperturbative methods, there are also immediate benefits, which present an advantage compared, e.g., to quark-model studies. An excellent example is the possibility to prove results that are exact in QCD. Prominently, chiral symmetry and its dynamical breaking, along with the corresponding constraints, are manifested via the axialvector Ward-Takahashi identity (AVWTI), which serves as a guide for the construction of consistent corresponding integration-equation kernels 25 27. Furthermore, the AVWTI provides insight on the properties of pseudoscalar mesons, which in the chiral limit reduces to the well-known Gell-Mann-Oakes-Renner relation, but can be formulated on general grounds. In a symmetrypreserving truncation such as the one used herein, these statements remain valid and can be checked also numerically. More precisely, our numerical studies of the pion and its radial excitations show the behavior that is exact in QCD in the chiral limit, namely a massless pion ground state with a finite decay constant and massive radially excited pion states with an exactly zero decay constant each 28, 29]. A similar situation is found with respect to electromagnetic properties, where the vector WTI, also satisfied in RL truncation, and its effects can be tested numerically via charge-conservation and the behavior of electromagnetic form factors [21, 30, 34].

Another important advantage is the manifest covari- 
ance of the DSBSE setup, regardless of the truncation used. It implies immediate usability of both the quark propagators as well as the covariant amplitudes obtained as solutions of the BSE in any calculation of transition amplitudes between hadrons or currents and dressed vertex functions. Among the benefits of the covariant fourdimensional setup, one also gets direct access to meson states with exotic quantum numbers already at the $q \bar{q}$ level. As another advantage, we mention the connection to perturbative QCD via the effective interaction discussed below.

Due to these advantages, the approach has been successfully applied to many individual problems in and beyond spectroscopy; concrete examples and therefore intrinsically relevant as outlook of this work are, apart from chiral and electromagnetic hadron properties already cited above, strong hadron decay widths [35, 36], valence-quark distributions of pseudoscalar mesons $37-$ 40], studies of tensor mesons [41, 42] and extensions of this setup to QCD at finite temperature [43 45].

While all these individual results and studies provide quite a wealth of information and a large portion was even computed with the same model (which is also used here), there is no comprehensive meson, let alone hadron study so far, and our work is the first step towards one. At the level of RL truncation, it remains to be shown what the range of success of such a comprehensive endeavor can be or whether it is possible at all. As a final part of motivation, it is helpful to mention that even a successful study of radial meson excitations such as the one presented herein has been generally doubted and deemed impossible, which makes our results relevant in the first place and remarkable at the same time.

We note at this point that our calculations have been performed using Landau-gauge QCD in Euclidean momentum space. Progress made using the Minkowskispace formulation of the BSE to study the $q \bar{q}$ system is ongoing and can be traced via [46 54. Calculations in the Coulomb gauge of QCD are slightly different in terms of numerical feasibility as well as the particular systems or domains that are more easily described. For details, see [15, 55, 60] and references therein.

The paper is organized as follows: in Sec. II we review the benefits and caveats of the rainbow-ladder (RL) truncation of the DSBSE system. Section III contains the details on the effective interaction used herein. Results and conclusions are presented in Secs. [V] and V] respectively.

\section{RAINBOW-LADDER TRUNCATION}

For comprehensive phenomenological modeling with a realistic effective interaction, the truncation of choice currently is the RL truncation of the quark-DSE-mesonBSE system.

Studies beyond-RL truncation are often exploratory in nature and use an interaction simple enough to deal with the complexity of particular aspects of the infinite tower of the DSEs and the corresponding BSE setup but too simple to retain all features required for a successful spectroscopy of hadrons, let alone the calculation of transition matrix elements [26, 27, 61].

In more sophisticated settings, the effective interaction is realistic overall or at least in some particular aspect of the diagrammatic setup of the truncation scheme 62 71. As an alternative, other studies have approached the problem of constructing a consistent BSE kernel for a given quark-gluon vertex on a more general footing, see [72, 73] and references therein. However, such investigations have never been comprehensive due to the numerical and conceptual difficulty involved. In addition, neither the conceptional problems of the BSE such as the determination of the analytic structure of the quark propagator or the possible spurious nature of some excited states, nor the phenomenological problems encountered, e. g., in the description of axial-vector meson states, were satisfactorily resolved. In this sense, an RL study can be considered reasonable and most notably constructive towards the goal of a comprehensive phenomenological application of the DSBSE approach.

It is important to note here again that RL truncation satisfies the relevant (axial-vector and vector) WardTakahashi identities (see e. g. [25, 28, 30, 74, 78]) and thus remains true to the underlying QCD in the corresponding respects. A reliable numerical setup (ours is detailed in [41, 79 81]) is important, in particular, with increasing quark mass.

\section{BOUND STATE EQUATION AND MODEL INTERACTION}

We note at this point that meson studies like ours can be conducted equally well using the homogeneous BSE or an analogous but more general inhomogeneous vertex BSE, see, e.g. 43, 82, 83. Herein we employ the homogeneous $q \bar{q} \mathrm{BSE}$ in $\mathrm{RL}$ truncation which reads

$$
\begin{aligned}
& \Gamma(p ; P)=-C_{F} \int_{q}^{\Lambda} \mathcal{G}\left((p-q)^{2}\right) D_{\mu \nu}^{\mathrm{f}}(p-q) \gamma_{\mu} \chi(q ; P) \gamma_{\nu} \\
& \chi(q ; P)=S\left(q_{+}\right) \Gamma(q ; P) S\left(q_{-}\right),
\end{aligned}
$$

where $q$ and $P$ are the quark-antiquark relative and total momenta, respectively, and the (anti)quark momenta are chosen as $q_{ \pm}=q \pm P / 2$. This equation requires knowledge of the quark propagator $S(p)$, which is obtained from its DSE ( $C_{F}=4 / 3$ is the Casimir color factor)

$$
\begin{aligned}
S(p)^{-1} & =\left(i \gamma \cdot p+m_{q}\right)+\Sigma(p) \\
\Sigma(p) & =C_{F} \int_{q}^{\Lambda} \mathcal{G}\left((p-q)^{2}\right) D_{\mu \nu}^{\mathrm{f}}(p-q) \gamma_{\mu} S(q) \gamma_{\nu} .
\end{aligned}
$$

In the above, the effective interaction is denoted by $\mathcal{G}$ and will be specified in detail below. $\Sigma$ is the quark selfenergy, $m_{q}$ is the current-quark mass, $D_{\mu \nu}^{\mathrm{f}}$ represents the 
free gluon propagator and $\gamma_{\nu}$ is the bare quark-gluon vertex's Dirac structure. Dirac and flavor indices are omitted for brevity. $\int_{q}^{\Lambda}=\int^{\Lambda} d^{4} q /(2 \pi)^{4}$ denotes a translationally invariant regularization of the integral, with the regularization scale $\Lambda$ [84].

The evolution of the RL effective interaction $\mathcal{G}$ started from a Dirac- $\delta$ in momentum space, which reduces the coupled integral equations to a set of coupled algebraic equations [85. For several studies on different levels of sophistication with regard to the numerical treatment of the evaluation of the quark-propagator dressing functions needed as input in the BSE, additions and modifications were made to this term such as a 2-loop perturbativeQCD contribution and an Ansatz for the infrared behavior [86, 87] as well as one-loop perturbative QCD together with modified [75, 88, 92] or additional strength in the intermediate-momentum regime both with 84 and without the $\delta$-term [35, 93 95]. Further modifications include a focus on low and intermediate momenta with less emphasis on the perturbative part 96] and even an ultra-violet (UV) finite version [97, which emphasized the importance of the intermediate-momentum domain for spectroscopy; in addition, it was shown that the influence of the far-infrared behavior of the interaction on meson properties is minor [98] for the concrete case of the $\rho$ meson mass and decay constant.

In addition, alternative approaches have been proposed, where the effective coupling is adjusted via the quark-gluon vertex such that the quark mass function remains independent on the normalization point, and the correct asymptotic behavior is preserved [99, 100]. Furthermore, models have been constructed for the effective interaction such that gauged lattice quark propagators are reproduced via the quark DSE solutions [101 105.

We also mention here that corrections to the RL truncation are expected to diminish with increasing quark mass, which prompted the investigations in [106, [107], where a quark-mass dependence was introduced in the main interaction parameters and the setup was tested for both meson and baryon states. Recently, more evidence towards the necessity of a quark-mass dependence of a phenomenologically successful RL study of meson properties has emerged [70]. In our present work and strategy, we include this possibility in a natural way, as is detailed below.

The model of Ref. 93 was used extensively in the past to investigate the meson spectrum and various meson properties with great success, in particular in the pseudoscalar and vector channels (see also the references given above) and this parameterization is our choice herein as well. It reads

$$
\frac{\mathcal{G}(s)}{s}=\frac{4 \pi^{2} D}{\omega^{6}} s \mathrm{e}^{-s / \omega^{2}}+\frac{4 \pi \gamma_{\mathrm{m}} \pi \mathcal{F}(s)}{1 / 2 \ln \left[\tau+\left(1+s / \Lambda_{\mathrm{QCD}}^{2}\right)^{2}\right]} .
$$

The first term is characterized by the parameters $\omega$ (which corresponds to an effective inverse range of the interaction) and $D$ (which acts like an overall strength) and determines the intermediate-momentum part of the interaction, while the second describes the UV and produces the correct one-loop perturbative QCD limit. $\mathcal{F}(s)=$ $\left[1-\exp \left(-s /\left[4 m_{\mathrm{t}}^{2}\right]\right)\right] / s$ where $m_{\mathrm{t}}=0.5 \mathrm{GeV}, \tau=\mathrm{e}^{2}-1$, $N_{\mathrm{f}}=4, \Lambda_{\mathrm{QCD}}^{N_{\mathrm{f}}=4}=0.234 \mathrm{GeV}$, and $\gamma_{\mathrm{m}}=12 /\left(33-2 N_{\mathrm{f}}\right)$, which is left unchanged from Ref. 93].

In addition to the current-quark masses, $\omega$ and $D$ are those parameters of the interaction whose impact on meson spectroscopy provides the focus of this work. It was found already in 93] that pseudoscalar- and vectormeson ground-state properties remained unchanged for light mesons if one varies $\omega$ in the range $[0.3,0.5] \mathrm{GeV}$ and determines $D$ by keeping their product fixed to the phenomenologically successful value of $D \times \omega=0.372$ $\mathrm{GeV}^{3}$. Essentially, this corresponds to the statement that ground states, which in the quark model have orbital angular momentum $l=0$, have properties that do not depend strongly on the effective range of the longrange (intermediate-momentum) piece of the strong effective interaction; this situation was contrasted by the case of radially excited meson states [32, 94, 108, and other types of excitations, most prominently those corresponding to $l \neq 0$ in the quark model [41, 109]. These dependences can be used to sufficiently constrain all parameters of the interaction, in particular both $\omega$ and $D$. In fact, the more states our model is compared to, the more difficult it is to achieve a decent overall description, which is a real challenge both for the model setup as well as for RL truncation itself.

After a recent quarkonium study (restricted to the $D \times \omega=$ const. prescription but still successful for the ground-states in bottomonium and, to some extent, also charmonium) was presented in [110, herein we require a more comprehensively successful description of experimental data, in particular including radially excited states in each $J^{P C}$ channel. To attempt such an agreement with experiment, we vary $\omega$ and $D$ independently along the lines of a strategy outlined in detail in [11], where this investigation was already carried out for bottomonium. In short, the parameters are fitted to a set of representative experimental level splittings first; in a second step, the quark mass is determined by a least-squares fit to the ground-state bottomonium masses known experimentally.

Here, we add the case of charmonium and discuss the consequences of our results for states with exotic quantum numbers as well as a number of states found experimentally, whose quantum numbers have not yet been determined completely. It is noteworthy that we fit the values of $\omega$ and $D$ separately for each current-quark mass, such that a quark-mass dependence of these parameters will emerge. The next section reviews the situation in bottomonium and details our charmonium results. 


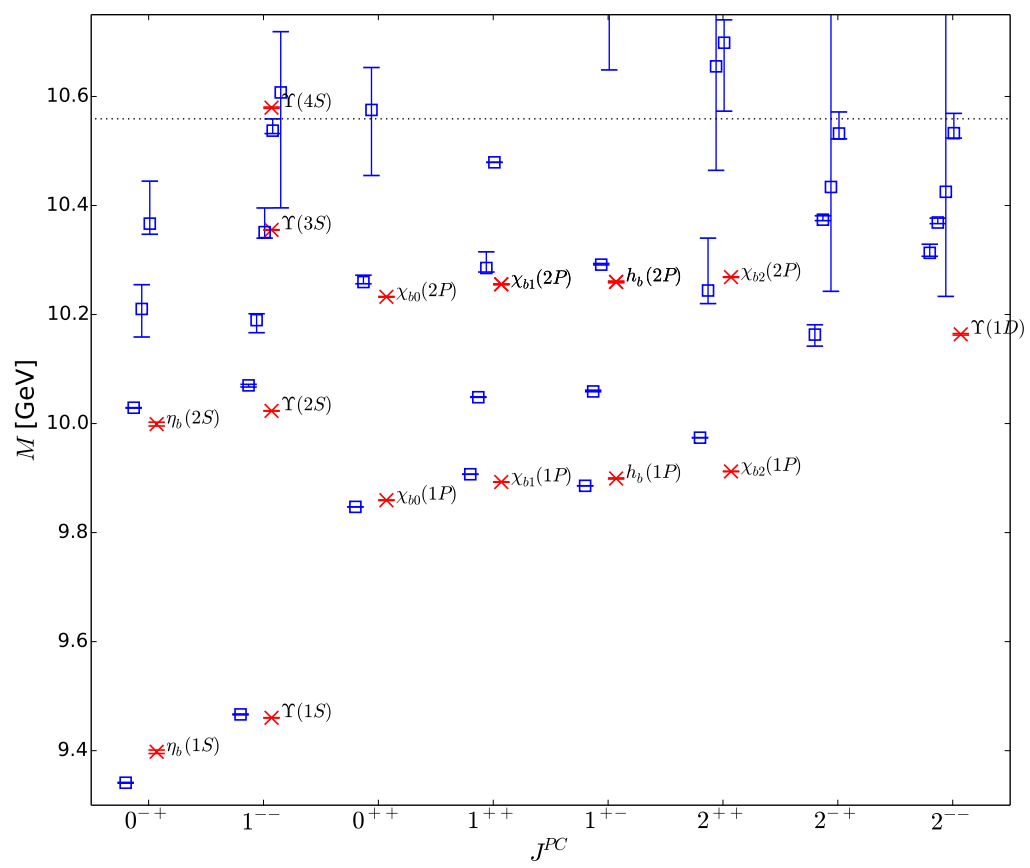

FIG. 1. (Color online) Bottomonium spectrum: calculated (blue boxes) versus experimental data (red crosses) [112]. Theoretical error bars represent uncertainties from extrapolation techniques, where necessary (see text). The horizontal dotted line marks the open-flavor threshold.

\section{RESULTS AND DISCUSSION}

In [11] we obtained our best fit to the bottomonium spectrum for $m_{\mathrm{b}}=3.635 \mathrm{GeV}$ (given at a renormalization point $\mu=19 \mathrm{GeV}$ ) together with $\omega=0.7 \mathrm{GeV}$ and $D=1.3 \mathrm{GeV}^{2}$. The results are shown as blue boxes in Fig. 1. together with experimental data [112, shown as red crosses. In the same way, we fitted the charmonium spectrum and obtained $m_{\mathrm{c}}=0.855 \mathrm{GeV}$ (given at a renormalization point $\mu=19 \mathrm{GeV}$ ) together with $\omega=0.7$ $\mathrm{GeV}$ and $D=0.5 \mathrm{GeV}^{2}$; the results are shown in Fig. 2. together with experimental data [112, 113. We note that our error bars, where relevant, come from extrapolated results in situations where propagator singularities prohibit a direct cacluation; details on the source of this problem and our extrapolation strategy can be found in the appendix as well as the appendices of [41, 110, 114].

In addition, it is important to note that our results correspond to bound states and not resonances due to the effect of the truncation: open hadronic decay channels are not contained in the RL-BSE interaction kernel. Hadronic (and other) decay width or properties are computed from the solutions of the BSE as well as the quarkpropagators in a semi-perturbative fashion. In particular, as mentioned in the introduction, efforts have been made towards the calculation of vector to pseudoscalarpseudoscalar decays for light and strange mesons 35] as well as the $\Delta$ in the baryon sector 36 . While it is both natural and desirable for our study to include such re- sults in the future, the effort to achieve them is clearly beyond the scope of the present manuscript. For the moment, we can only caution the reader when comparing our results to experimental data above the respective open-flavor thresholds. Apparently, this issue is of lesser importance in the bottomonium case than for charmonium. To illustrate this and to facilitate the analysis of our results we have marked the thresholds in our figures by horizontal dotted lines.

We begin the discussion with bottomonium shown in Fig. 1. where we find very good agreement between our results and well-established experimental data. Most splittings are well reproduced, in particular between ground and radially excited states in each channel. It is noteworthy that we find the correct level ordering of the first radially excited $0^{-+}$and $1^{--}$states in the bottomonium system; in a similar fashion, level orderings are well reproduced with a few exceptions. In general there is a clear identification of each ground- and first radially excited state known experimentally with one of our results. However, there are a few caveats. While a slight mismatch for the $2^{--}$ground state is apparent, we expect on the basis of [110] that this can be cured by further fine-tuning of model parameters. Higher radial excitations than the first are mostly unclear at the moment due to both theoretical and experimental uncertainties overall, except for the vector channel, where the experimental situation is excellent due to the prominent coupling to $e^{+} e^{-}$. We find excellent agreement for 


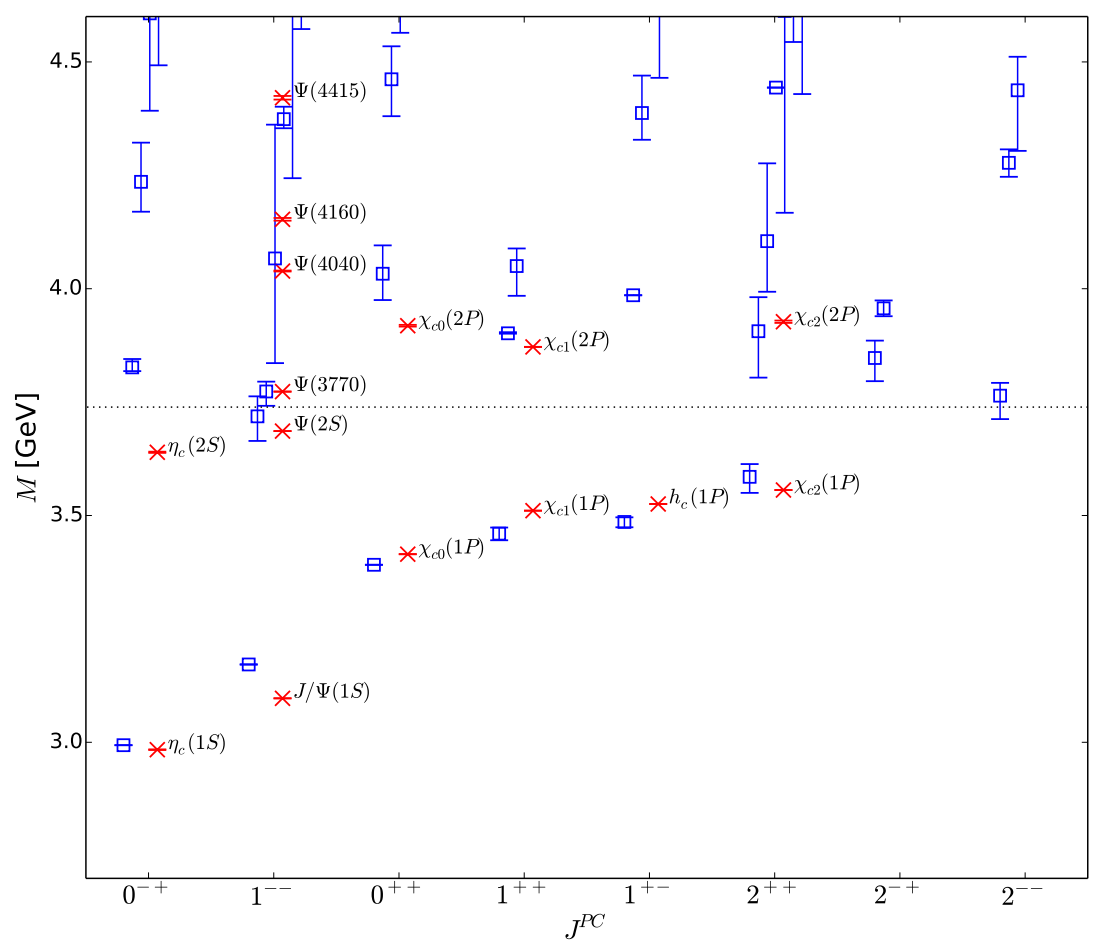

FIG. 2. (Color online) Charmonium spectrum: calculated (blue boxes) versus experimental data (red crosses) 112, 113. Theoretical error bars represent uncertainties from extrapolation techniques, where necessary (see text). The horizontal dotted line marks the open-flavor threshold.

the $\Upsilon(3 S)$, but at the same time one lower result without an experimental match. Further investigations are needed to clarify the role of this state, and are currently on their way. A similar situation is encountered in the axial-vector channels, where one extra calculated result each appears in between the ground and first radially excited experimental state. At the present time however, we have no reliable tools at hand to to determine, e. g., whether or not these extra states might be spurios solutions of the BSE. Consequently, we have to defer a more in-depth discussion to a later time. In the meantime, similarly to the vector case, we will use means beyond spectroscopy to determine the role of these states and report the results in future publications.

For charmonium presented in Fig. 2 the stateidentification between experiment and calculation is even better and much clearer: no extra calculated states are encountered in the domain of the ground states and first radial excitations. Again, splittings between radially excited and ground states in each channel are very well reproduced; the same is true for the level orderings with the exception of the $\eta_{c}(2 S)$, which is too heavy in our study. In addition to this excellent overall agreement, it is most notable, how closely the radial excitations in the vector channel can be matched, even beyond the $\Psi(2 S)$. With regard to the matching and quality of the description of experimental data in both bottomonium and charmonium, we note again that our search for the optimal model parameters was carried out within the setup of the particular model chosen. We expect that better agreement can be reached by further fine-tuning of the shape of the model interaction.

While we defer a detailed discussion of exotic-state masses in the various $J^{P C}$ channels to future works, we give a brief outlook already at this point: states with exotic quantum numbers are generally low in our RL study compared to expectations from other approaches. More concretely, we find the $0^{--}$and $1^{-+}$to be lowest in both bottomonium and charmonium. In the former case, they lie even below the $l=1$ ground states at $\sim 9.7 \mathrm{GeV}$, while in the latter they lie in the same region as the $l=1$ ground states at $\sim 3.6 \mathrm{GeV}$.

Before concluding, we present some evidence as to how feasible a description of both charmonium and bottomonium is with the same set of model parameters. To illustrate this, we present two more figures analogous to Figs. 1 and 2 , but with the other set of the model parameters $\omega$ and $D$, respectively. Thus, the bottomonium spectrum with $\omega=0.7 \mathrm{GeV}$ and $D=0.5 \mathrm{GeV}^{2}$ is shown in Fig. 3 and the charmonium spectrum with $\omega=0.7$ $\mathrm{GeV}$ and $D=1.3 \mathrm{GeV}^{2}$ is shown in Fig. 4. We observe that in both cases the good description of level orderings, radial splittings, and identification of states is destroyed. 


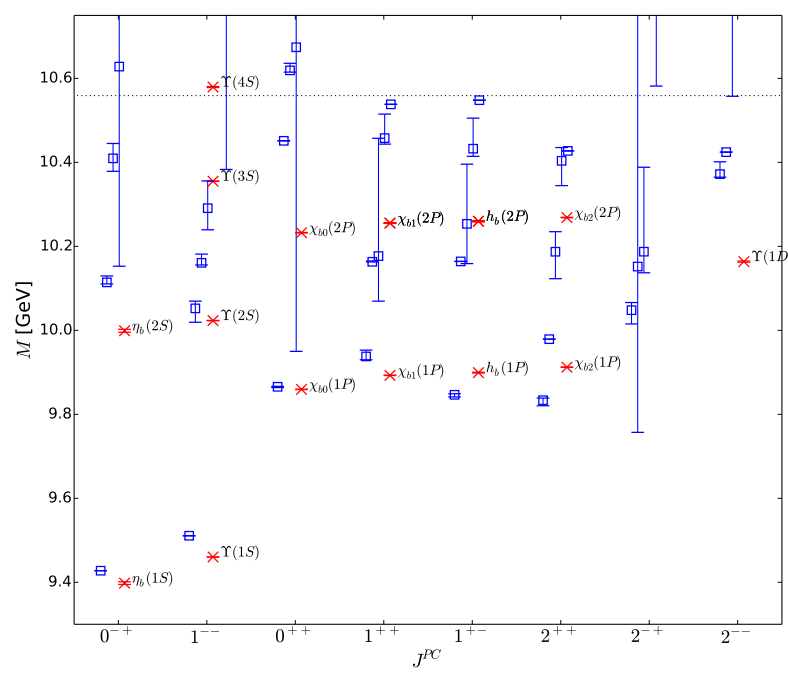

FIG. 3. (Color online) Bottomonium spectrum cross-check: Same style as Fig. 1. but computed with the optimal parameters from charmonium (see text).

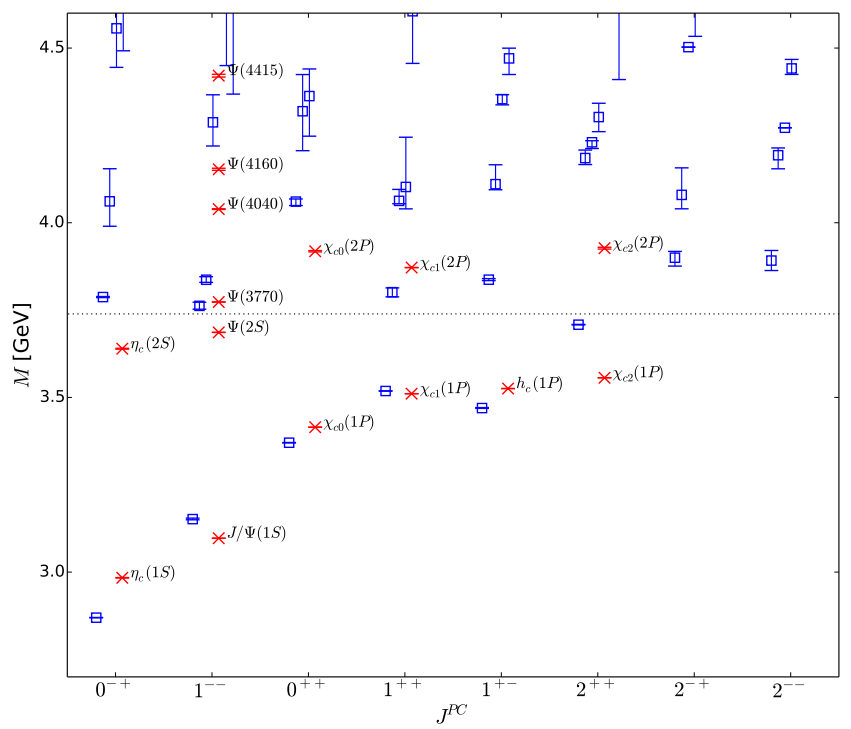

FIG. 4. (Color online) Charmonium spectrum cross-check: Same style as Fig. 2, but computed with the optimal parameters from bottomonium (see text).

\section{CONCLUSIONS AND OUTLOOK}

It has been speculated in the past how successful a comprehensive RL truncated DSBSE model of hadrons, or at least mesons, can be. The negligence of all but the $\gamma_{\mu}$ component of the quark-gluon vertex was generally believed to be too drastic as to still allow for a reasonable phenomenological description of the various splittings in the meson spectrum. These expectations were based on caclulations done mainly with light quarks and on a domain of the model parameters restricted by an- choring them in the light quark domain. In particular, primary anchors were the pion mass and decay constant as well as the chiral condensate. However, in this respect it is very important to differentiate between the light- and heavy-quark cases, since corrections to RL truncation are expected to diminish with increasing quark mass. Therefore, only an RL study anchored in the heavy-quark domain can be expected to be well-suited for comprehensive purposes. In addition, a quark-mass dependence in the effective interaction may provide a better way towards a unified description of meson spectra over the entire range of experimentally available quark masses.

In order to fully establish the DSBSE framework as an adequate and valuable complementary alternative to the quark model and other non-perturbative approaches to QCD, it is imperative to attempt a comprehensive study of hadrons. As a first step, the requirements for such a study must be taken beyond a collection of individual results towards a unified model study with as wide a scope as possible. We have identified an RL truncated DSBSE setup with a sophisticated model interaction as a candidate for such a study and presented the first step here. In our study of heavy quarkonia we have determined the sets of model parameters that optimize an RL DSBSE description of the meson spectrum, including both ground states and radial excitations for the first time. We found good overall agreement with experimental data to a degree well beyond the general expectations regarding the truncation used herein. Nonetheless, there are caveats, in particular extra states in the vector $\left(1^{--}\right)$and axialvector $\left(1^{++}\right.$and $\left.1^{+-}\right)$channels in bottomonium as well as a lack of clarity in the computational outcome for the higher radial excitations, both of which are subject of ongoing further studies.

The next steps are to extend this study to the lightquark sector, investigate the role of extra states in the calculated results as well as attempt to identify experimental states with undetermined quantum numbers or some of the $X, Y$, and $Z$ states, respectively, with appropriate results from our calculations. The set of results will include masses and decay constants at first, and later also comprise electromagnetic as well as hadronic width and properties. We emphasize that this includes to present and discuss concrete results for states with exotic quantum numbers. In the course of our studies, we may allow even more free parameters or a different functional form in the effective interaction, whose parametric degrees of freedom have not yet been fully exploited, in order to more effectively fine-tune the results, if necessary.

\section{ACKNOWLEDGMENTS}

We acknowledge helpful conversations with M. Blank, G. Eichmann, and R. Williams. This work was supported by the Austrian Science Fund (FWF) under project no. P25121-N27. 


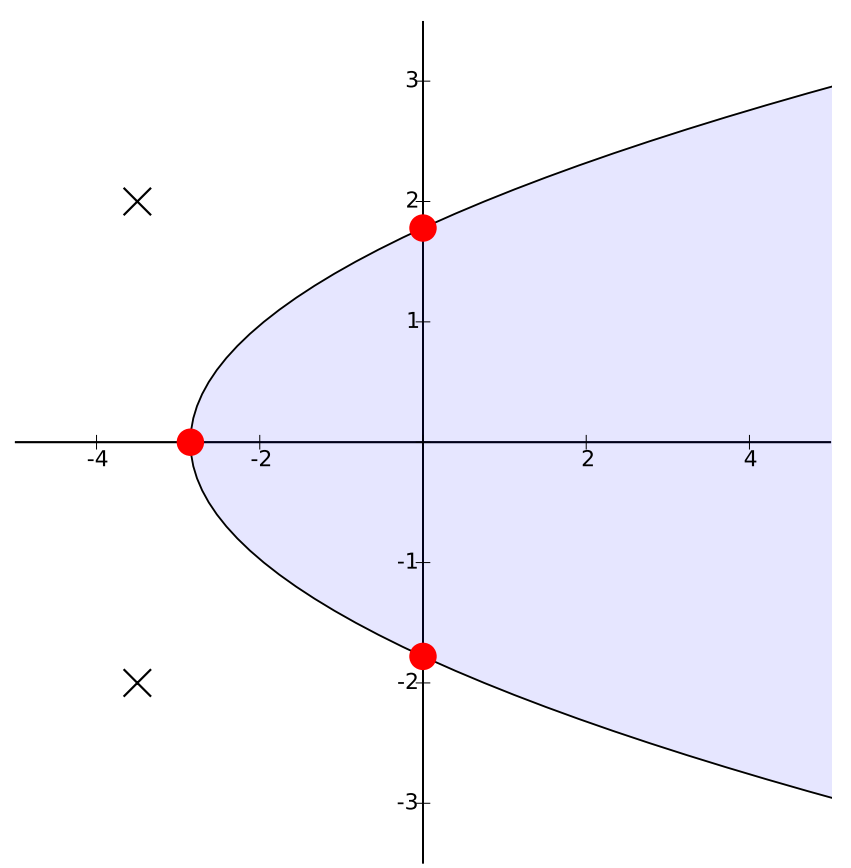

FIG. 5. (Color online) Integration domain (light blue area) with parabolic boundary in the complex $q_{ \pm}^{2}$-plane, on which the (anti)quark propagator dressing functions need to be known numerically. The red dots identify the intersection points with the real and imaginary axes; the crosses illustrate the typical location of singularities in the dressing functions limiting the integration domain (see text).

\section{Appendix A: Technicalities}

In the Euclidean-space formulation of the DSBSE approach to mesons, the BSE contains two dressed (anti)quark propagators that depend on the momenta $q_{ \pm}$ as given in Eq. (1) and below. With a timelike total momentum $P$ and the integration momentum $q$ being the gluon momentum, one needs to compute the propagator dressing functions in a region of the complex $q_{ \pm}^{2}$-plane that lies inside a parabolic boundary, stretching towards real positive infinity, indicated as the light blue area in Fig. 5. Assuming a real, positive bound-state mass of $M$ with $P^{2}=-M^{2}$ and two equal-mass constituents, the corresponding integration domain can be defined via the three intersection points of the parabolic boundary with the real and imaginary axes, at $\left(-M^{2} / 4,0\right)$ and $\left(0, \pm M^{2} / 2\right)$, respectively, marked by the red dots in Fig. 5. In practice, keeping the numerical setup straightforward [80, this means that any singular structure in the propagator dressing functions puts a limit on the maximum bound-state mass obtainable via standard methods; a typical scenario is depicted in Fig. 5. where singularity positions are marked with black crosses. While a ground-state calculation is mostly safe from such problems, excited states mostly lie above the mass range obtainable directly. As a simple way to deal with this, one can resort to extrapolation techniques. First steps had

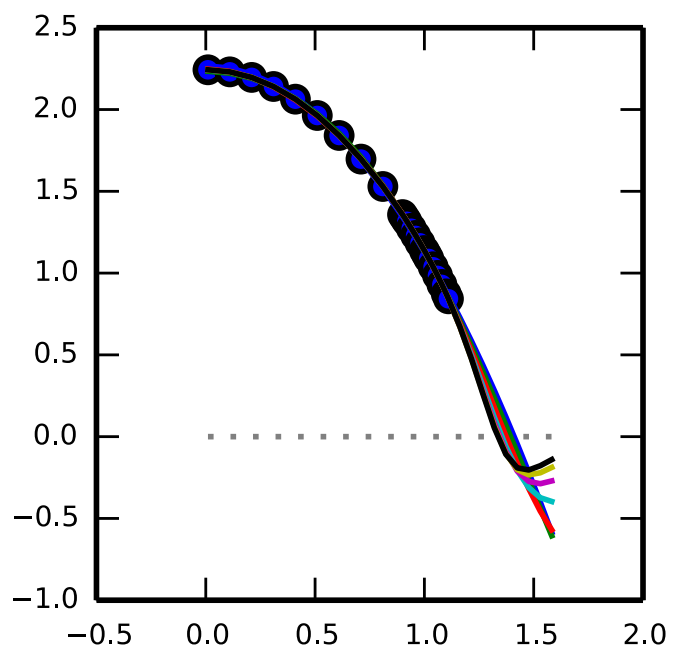

FIG. 6. (Color online) Example for extrapolation of $\tilde{\lambda}\left(P^{2}\right)$ curves with different $N$ represented by different colors to obtain the bound state mass $M$ where $\tilde{\tilde{\lambda}}\left(P^{2}\right)=0$, as defined in Eq. A2 and below, for a pseudoscalar radial excitation.

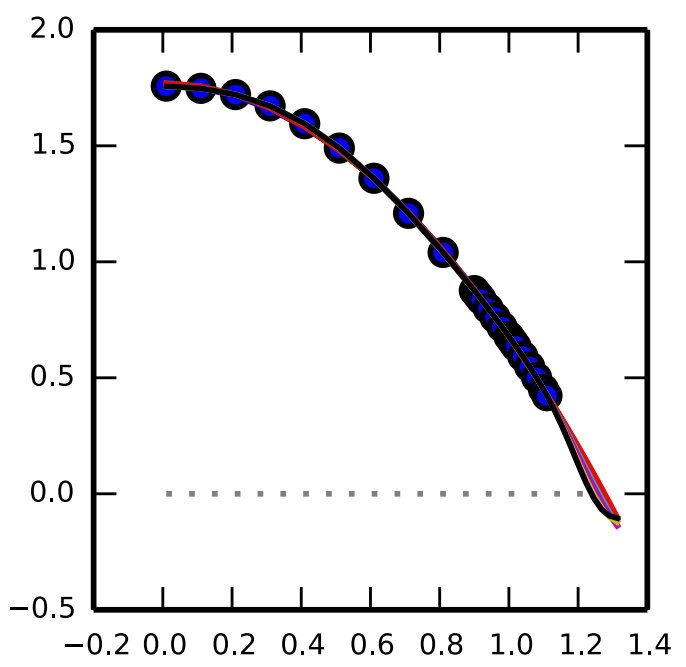

FIG. 7. (Color online) Same as Fig. 6 for a scalar radial excitation.

been taken in [41] and a more sophisticated setup has been used in [110] and also herein. As a result, the extrapolation introduces an uncertainty in our calculation, which we acknowledge by plotting error bars on our resulting masses. To immediately illustrate typical cases, we present extrapolations for a pseudoscalar and a scalar radially excited case in Figs. 6 and 7 respectively. To understand the curves shown in these figures, consider the homogeneous BSE as a $P^{2}$-dependent eigenvalue equation of the form

$$
\lambda\left(P^{2}\right) \Gamma\left(P^{2}\right)=\int K S\left(P^{2}\right) \Gamma\left(P^{2}\right) S\left(P^{2}\right),
$$

where the original BSE is recovered for the eigenvalue $\lambda\left(P^{2}\right)=1$ (for more details, see [80]). In this fashion, in- 
formation about ground- and excited-state solutions can be extracted also off-shell and then extrapolated to the on-shell point. We use, as provided in [110, the form

$$
\tilde{\lambda}\left(P^{2}\right):=\frac{\lambda\left(P^{2}\right)}{1-\lambda\left(P^{2}\right)}=\frac{r}{P^{2}+M^{2}}+\sum_{i=1}^{N}\left(P^{2}\right)^{i} c_{i}
$$

to fit our results for the eigenvalues $\lambda$ obtained on a reasonable and directly accessible range of $P^{2}$ and straightforwardly obtain the bound-state mass $M$ as well as the other fit constants $r$ and $c_{i}$. To understand the figures, it is important to note that, in order to reach $\lambda\left(P^{2}\right)=1$, we require that $\tilde{\lambda}\left(P^{2}\right)=0$. The fits are repeated with different numbers of correction terms $N$ in Eq. A2, where we ensure that the fit results remain stable by a reasonable choice of the maximum value of $N$. Different values of $N$ yield the differently colored curves in Figs. 6 and 7 , while our calculated points are depicted by black circles and the dotted line marks zero. We use the arithmetic mean as our final result and the differences to the largest and smallest values as the upper and lower error bars, as they are given in Figs. 1- 4 .
[1] D. J. Gross and F. Wilczek, Phys. Rev. Lett. 30, 1343 (1973).

[2] D. J. Gross and F. Wilczek, Phys. Rev. D 8, 3633 (1973)

[3] H. D. Politzer, Phys. Rev. Lett. 30, 1346 (1973)

[4] N. Brambilla, S. Eidelman, P. Foka, S. Gardner, A. Kronfeld, et al., 1404.3723 [hep-ph]

[5] J. J. Dudek, R. G. Edwards, N. Mathur, and D. G. Richards, Phys. Rev. D 77, 034501 (2008).

[6] L. Liu et al. (Hadron Spectrum Collaboration), JHEP 1207, $126(2012)$

[7] C. Thomas, PoS LATTICE2013, 003 (2013).

[8] W. Lucha, D. Melikhov, and S. Simula, Phys.Lett. B735, 12 (2014)

[9] J. Wambach, R.-A. Tripolt, N. Strodthoff, and L. von Smekal, 1404.7312 [hep-ph]

[10] J. M. Pawlowski, Annals Phys. 322, 2831 (2007)

[11] S. J. Brodsky, G. F. de Teramond, H. G. Dosch, and J. Erlich, 1407.8131 [hep-ph].

[12] C. D. Roberts, 1203.5341 [nucl-th]

[13] A. Bashir, L. Chang, I. C. Cloet, B. El-Bennich, Y.-X. Liu, et al., Commun.Theor.Phys. 58, 79 (2012),

[14] C. Popovici, Mod.Phys.Lett. A28, 1330006 (2013).

[15] C. Popovici, P. Watson, and H. Reinhardt, Phys. Rev. D 81, 105011 (2010).

[16] C. S. Fischer, A. Maas, and J. M. Pawlowski, Annals Phys. 324, 2408 (2008).

[17] J. C. R. Bloch, A. Krassnigg, and C. D. Roberts, FewBody Syst. 33, 219 (2003).

[18] G. Eichmann, A. Krassnigg, M. Schwinzerl, and R. Alkofer, Annals Phys. 323, 2505 (2008)

[19] G. Eichmann, R. Alkofer, A. Krassnigg, and D. Nicmorus, Phys. Rev. Lett. 104, 201601 (2010).

[20] H. Sanchis-Alepuz, G. Eichmann, S. Villalba-Chavez, and R. Alkofer, Phys.Rev. D84, 096003 (2011).

[21] G. Eichmann and C. S. Fischer, Phys.Rev. D87, 036006 (2013)

[22] J. Segovia, C. Chen, I. C. Cloet, C. D. Roberts, S. M. Schmidt, et al., Few Body Syst. 55, 1 (2014).

[23] H. Sanchis-Alepuz, R. Williams, and R. Alkofer, Phys.Rev. D87, 096015 (2013)

[24] H. Sanchis-Alepuz and C. S. Fischer, 1408.5577 [hep$\mathrm{ph}]$

[25] H. J. Munczek, Phys. Rev. D 52, 4736 (1995)

[26] A. Bender, C. D. Roberts, and L. Von Smekal, Phys. Lett. B 380, 7 (1996)
[27] M. S. Bhagwat, A. Holl, A. Krassnigg, C. D. Roberts, and P. C. Tandy, Phys. Rev. C 70, 035205 (2004)

[28] P. Maris, C. D. Roberts, and P. C. Tandy, Phys. Lett. B 420, 267 (1998).

[29] A. Holl, A. Krassnigg, and C. D. Roberts, Phys. Rev. C 70, 042203(R) (2004)

[30] P. Maris and P. C. Tandy, Phys. Rev. C 61, 045202 (2000).

[31] P. Maris and P. C. Tandy, Nucl. Phys. Proc. Suppl. 161, $136(2006)$.

[32] A. Holl, A. Krassnigg, P. Maris, C. D. Roberts, and S. V. Wright, Phys. Rev. C 71, 065204 (2005)

[33] M. S. Bhagwat and P. Maris, Phys. Rev. C 77, 025203 (2008).

[34] G. Eichmann, Phys. Rev. D 84, 014014 (2011).

[35] D. Jarecke, P. Maris, and P. C. Tandy, Phys. Rev. C 67, $035202(2003)$.

[36] V. Mader, G. Eichmann, M. Blank, and A. Krassnigg, Phys. Rev. D 84, 034012 (2011).

[37] T. T. Nguyen, Aspects of non-perturbative QCD for meson physics, Ph.D. thesis, Kent State University (2010).

[38] P. C. Tandy, AIP Conf.Proc. 1374, 139 (2011)

[39] T. Nguyen, A. Bashir, C. D. Roberts, and P. C. Tandy, Phys. Rev. C83, 062201 (2011).

[40] L. Chang, I. Cloet, C. Roberts, S. Schmidt, and P. Tandy, Phys.Rev.Lett. 111, 141802 (2013)

[41] A. Krassnigg and M. Blank, Phys. Rev. D 83, 096006 (2011).

[42] C. S. Fischer, S. Kubrak, and R. Williams, 1406.4370 [hep-ph],

[43] P. Maris, C. D. Roberts, S. M. Schmidt, and P. C. Tandy, Phys. Rev. C 63, 025202 (2001)

[44] D. Horvatic, D. Klabucar, and A. E. Radzhabov, Phys. Rev. D 76, 096009 (2007)

[45] M. Blank and A. Krassnigg, Phys. Rev. D 82, 034006 (2010).

[46] V. Sauli, J. Phys. G 35 (2008).

[47] J. Carbonell and V. Karmanov, Eur.Phys.J. A46, 387 (2010)

[48] V. Sauli, Phys.Rev. D86, 096004 (2012)

[49] T. Frederico, G. Salme, and M. Viviani, Phys.Rev. D85, 036009 (2012)

[50] V. Sauli, Phys.Rev. D90, 016005 (2014).

[51] J. Carbonell and V. Karmanov, Phys.Lett. B727, 319 (2013) 
[52] T. Frederico, G. Salmé, and M. Viviani, 1312.0521 [hep-ph].

[53] J. Carbonell and V. Karmanov, 1408.3761 [hep-ph]

[54] R. L. Hall and W. Lucha, 1408.6330 [math-ph]

[55] R. Alkofer, M. Kloker, A. Krassnigg, and R. F. Wagenbrunn, Phys. Rev. Lett. 96, 022001 (2006)

[56] M. Gomez-Rocha, F. J. Llanes-Estrada, D. Schutte, and S. Villalba-Chavez, Eur.J.Phys. A44, 411 (2010)

[57] F. J. Llanes-Estrada et al., Fizika B20, 63 (2011).

[58] S. R. Cotanch and F. J. Llanes-Estrada, Fizika B20, 1 (2011).

[59] C. Popovici, P. Watson, and H. Reinhardt, Phys. Rev. D83, 125018 (2011).

[60] C. Popovici, Quark sector of Coulomb gauge Quantum Chromodynamics, Ph.D. thesis, University of Tuebingen (2011), 1106.0691 [hep-ph],

[61] M. Gomez-Rocha, T. Hilger, and A. Krassnigg, 1408.1077 [hep-ph]

[62] P. Watson and W. Cassing, Few-Body Syst. 35, 99 (2004).

[63] P. Watson, W. Cassing, and P. C. Tandy, Few-Body Syst. 35, 129 (2004)

[64] C. S. Fischer, P. Watson, and W. Cassing, Phys. Rev. D 72, 094025 (2005).

[65] H. H. Matevosyan, A. W. Thomas, and P. C. Tandy, Phys. Rev. C 75, 045201 (2007).

[66] H. H. Matevosyan, A. W. Thomas, and P. C. Tandy, J. Phys. G 34, 2153 (2007)

[67] C. S. Fischer and R. Williams, Phys. Rev. D 78, 074006 (2008)

[68] C. S. Fischer and R. Williams, Phys. Rev. Lett. 103, 122001 (2009)

[69] R. Williams, EPJ Web Conf. 3, 03005 (2010)

[70] R. Williams, 1404.2545 [hep-ph]

[71] H. Sanchis-Alepuz, C. S. Fischer, and S. Kubrak, 1401.3183 [hep-ph]

[72] L. Chang and C. D. Roberts, Phys. Rev. Lett. 103, 081601 (2009)

[73] W. Heupel, T. Goecke, and C. S. Fischer, Eur.Phys.J. A50, 85 (2014).

[74] T. Maskawa and H. Nakajima, Prog. Theor. Phys. 52, 1326 (1974)

[75] K.-I. Aoki, M. Bando, T. Kugo, M. G. Mitchard, and H. Nakatani, Prog. Theor. Phys. 84, 683 (1990),

[76] T. Kugo and M. G. Mitchard, Phys. Lett. B 282, 162 (1992).

[77] M. Bando, M. Harada, and T. Kugo, Prog. Theor. Phys. 91, 927 (1994)

[78] P. Maris and P. C. Tandy, Phys. Rev. C 62, 055204 (2000).

[79] A. Krassnigg, PoS Confinement8, 075 (2008).

[80] M. Blank and A. Krassnigg, Comput. Phys. Commun. 182, 1391 (2011)

[81] M. Blank, Properties of quarks and mesons in the Dyson-Schwinger/Bethe-Salpeter approach, Ph.D. thesis, University of Graz (2011), 1106.4843 [hep-ph].

[82] M. S. Bhagwat, A. Hoell, A. Krassnigg, C. D. Roberts, and S. V. Wright, Few-Body Syst. 40, 209 (2007).

[83] M. Blank and A. Krassnigg, AIP Conf. Proc. 1343, 349 (2011)
[84] P. Maris and C. D. Roberts, Phys. Rev. C 56, 3369 (1997).

[85] H. J. Munczek and A. M. Nemirovsky, Phys. Rev. D 28, $181(1983)$

[86] H. J. Munczek and P. Jain, Phys. Rev. D 46, 438 (1992)

[87] P. Jain and H. J. Munczek, Phys. Rev. D 48, 5403 (1993)

[88] J. L. Richardson, Phys.Lett. B82, 272 (1979)

[89] K. Higashijima, Phys.Rev. D 29, 1228 (1984).

[90] K. Higashijima, Prog.Theor.Phys.Suppl. 104, 1 (1991).

[91] T. Kugo and M. G. Mitchard, Phys. Lett. B 286, 355 (1992)

[92] N. Yamanaka, T. M. Doi, S. Imai, and H. Suganuma, Phys.Rev. D 88, 074036 (2013)

[93] P. Maris and P. C. Tandy, Phys. Rev. C 60, 055214 (1999)

[94] S.-X. Qin, L. Chang, Y.-X. Liu, C. D. Roberts, and D. J. Wilson, Phys.Rev. C85, 035202 (2012).

[95] C. S. Fischer, S. Kubrak, and R. Williams, 1409.5076 [hep-ph]

[96] M. R. Frank and C. D. Roberts, Phys. Rev. C 53, 390 (1996)

[97] R. Alkofer, P. Watson, and H. Weigel, Phys. Rev. D 65, $094026(2002)$

[98] M. Blank, A. Krassnigg, and A. Maas, Phys. Rev. D 83, 034020 (2011).

[99] C. S. Fischer and R. Alkofer, Phys. Rev. D 67, 094020 (2003).

[100] R. Alkofer, C. S. Fischer, and R. Williams, Eur. Phys. J. A 38, 53 (2008).

[101] M. S. Bhagwat, M. A. Pichowsky, C. D. Roberts, and P. C. Tandy, Phys. Rev. C 68, 015203 (2003).

[102] A. Krassnigg and C. D. Roberts, Nucl. Phys. A 737, 7 (2004)

[103] A. Krassnigg and C. D. Roberts, Fizika B 13, 143 (2004).

[104] C. S. Fischer and M. R. Pennington, Phys. Rev. D 73, 034029 (2006).

[105] G. Eichmann, A. Krassnigg, M. Schwinzerl, and R. Alkofer, Prog. Part. Nucl. Phys. 61, 84 (2008)

[106] G. Eichmann, R. Alkofer, I. C. Cloet, A. Krassnigg, and C. D. Roberts, Phys. Rev. C 77, 042202(R) (2008).

[107] G. Eichmann, I. C. Cloet, R. Alkofer, A. Krassnigg, and C. D. Roberts, Phys. Rev. C 79, 012202(R) (2009).

[108] A. Holl, A. Krassnigg, C. D. Roberts, and S. V. Wright, Int. J. Mod. Phys. A 20, 1778 (2005)

[109] A. Krassnigg, Phys. Rev. D 80, 114010 (2009).

[110] M. Blank and A. Krassnigg, Phys. Rev. D 84, 096014 (2011)

[111] C. Popovici, T. Hilger, M. Gomez-Rocha, and A. Krassnigg, 1407.7970 [hep-ph].

[112] K. A. Olive and others (Particle Data Group), Chin. Phys. C38, 090001 (2014).

[113] N. Brambilla et al., Eur. Phys. J. C71, 1534 (2010)

[114] S. M. Dorkin, L. P. Kaptari, T. Hilger, and B. Kampfer, Phys.Rev. C89, 034005 (2014). 